\title{
DIFFERENTIAL EQUATIONS IN WHICH THE POISSON PROCESS PLAYS A ROLE
}

\author{
BY STANLEY KAPLAN
}

Communicated by Lipman Bers, November 21, 1963

1. Introduction. The telegrapher's equation

$$
\frac{\partial^{2} u}{\partial t^{2}}+2 a \frac{\partial u}{\partial t}=c^{2} \frac{\partial^{2} u}{\partial x^{2}}
$$

where $a$ and $c^{2}$ are positive constants, is treated in [1], as follows. Let $N(t)$ be the Poisson process with constant intensity $a$ (see below). Denote by $E\{\cdot\}$ expected values with respect to this process, and by $T(t)$ the random time defined by

$$
T(t)=\int_{0}^{t}(-1)^{N(\tau)} d \tau .
$$

Then, if $v(x, t)$ is any smooth solution of the wave equation

$$
\frac{\partial^{2} v}{\partial t^{2}}=c^{2} \frac{\partial^{2} v}{\partial x^{2}}
$$

the function $u(x, t)=E\{v(x, T(t))\}$ satisfies (1), with

$$
\lim _{t \rightarrow 0+} u(x, t)=v(x, 0)
$$

and

$$
\lim _{t \rightarrow 0+} \frac{\partial u}{\partial t}(x, 0)=\frac{\partial v}{\partial t}(x, 0) .
$$

Kac observes that this is the restatement, in the language of continuous stochastic processes, of a result of Goldstein [2], who, starting with a Poisson-type random walk, asymptotically obtained solutions of (1). The proof in [1] goes via direct computation, which may be done also in the case of the Laplacian in several space dimensions. That some sort of similar result must hold in even greater generality, and with a more elegant proof, was suggested by Professor $\mathrm{Kac}$, to whom the author is indeed grateful. The purpose of this note is to present such a result.

2. The Poisson process. Given a non-negative, continuous function $a(t)$, defined on $[0, \infty)$, one may associate with it a Markov 
process, called the "Poisson process with intensity $a$ " as follows: Our process, which we write $N(t)$ for short, starts at zero, i.e., $N(0)=0$, and, for $t \geqq s$, we have

$$
P\{N(t)-N(s)=m\}= \begin{cases}0 & \text { if } m<0, \\ \frac{\left[\int_{s}^{t} a(\tau) d \tau\right]^{m}}{m !} \exp \left[-\int_{s}^{t} a(\tau) d \tau\right] & \text { if } m \geqq 0, \\ 1 & \text { if } m=0 \text { and } t=s .\end{cases}
$$

As in [3, pp. 159-160], $N(t)$ may be considered as a random variable, i.e., $N(t)=N(t, \omega), \omega \in \Omega$, where $\Omega$ is the set of all functions $\omega: t \rightarrow \omega(t)$ $=N(t, \omega)$, from $[0, \infty)$ to the non-negative integers, which are zero for $t=0$, continuous from the right, and nondecreasing, and which have only finitely many discontinuities in each finite $t$ interval. Thus $P$ is a probability measure on $(\Omega, Q)$, where $Q$ is the $\sigma$-algebra generated by all sets of the form $\{\omega \mid N(t, \omega)=k\}$ for $t \geqq 0, k$ a non-negative integer; $E\{\cdot\}$ is the associated expectation operator. Finally, for our process $N(t)$, we define the random variable $T(t)=T(t, \omega)$ as in (2).

3. The basic identity. We now prove the following:

Lemma. Let $v(t)$ be twice continuously differentiable in $(-\tau, \tau)$. If we define

$$
u(t)=E\{v(T(t))\}
$$

then $u(t)$ satisfies

$$
u^{\prime \prime}(t)+2 a(t) u^{\prime}(t)=E\left\{v^{\prime \prime}(T(t))\right\}, \quad 0<t<\tau,
$$

with

$$
\lim _{t \rightarrow 0+} u(t)=v(0)
$$

and

$$
\lim _{t \rightarrow 0+} u^{\prime}(t)=v^{\prime}(0) .
$$

Proof. Since $|T(t)| \leqq t$, the second assertion is immediate. For each $\omega, T$ is an absolutely continuous function of $t$, and $\partial T / \partial t$ is bounded from above in absolute value by 1 , and approaches 1 as $t \rightarrow 0+$. By the chain rule, we obtain

$$
u^{\prime}(t)=E\left\{v^{\prime}(T(t)) \frac{\partial T}{\partial t}(t)\right\} .
$$


Applying the bounded convergence theorem, the last assertion follows. Finally, we observe that (4) is now equivalent to the linear integral identity

$$
\begin{aligned}
u(t)= & v(0)+v^{\prime}(0) \int_{0}^{t} \exp \left[-2 \int_{0}^{\tau} a(s) d s\right] d \tau \\
& +\int_{0}^{t} d \tau \int_{0}^{\tau} \exp \left[-2 \int_{\theta}^{\tau} a(s) d s\right] E\left\{v^{\prime \prime}(T(\theta))\right\} d \theta .
\end{aligned}
$$

By the Weierstrass approximation theorem, it suffices to prove (5) for $v(t)=t^{k}, k=0,1,2, \cdots$. For $k=0,(5)$ is immediate. For $k=1$,

$$
u(t)=E\{T(t)\}=\int_{0}^{t} E\left\{(-1)^{N(\tau)}\right\} d \tau
$$

But

$$
E\left\{(-1)^{N(\tau)}\right\}=\sum_{k=0}^{\infty}(-1)^{k} P\{N(\tau)=k\}=\exp \left[-2 \int_{0}^{\tau} a(s) d s\right]
$$

Thus, it remains to prove (5) for $k \geqq 2$. If we introduce

$$
\sigma_{k}(t)=\frac{1}{k !} E\left\{T^{k}(t)\right\}
$$

we have, by Fubini's theorem again,

$$
\sigma_{k}(t)=\int_{0}^{t} d \tau_{k} \int_{0}^{\tau_{k}} d \tau_{k-1} \cdots \int_{0}^{\tau_{2}} d \tau_{1} E\left\{(-1)^{N\left(\tau_{1}\right)+\cdots+N\left(\tau_{k}\right)}\right\} .
$$

But, if $\tau_{1} \leqq \tau_{2} \leqq \cdots \leqq \tau_{k+2}$, we have

$$
\begin{aligned}
E\left\{(-1)^{N\left(\tau_{1}\right)+\cdots+N\left(\tau_{k+2}\right)}\right\} & \\
= & E\left\{(-1)^{N\left(\tau_{1}\right)+\cdots+N\left(\tau_{k}\right)}(-1)^{N\left(\tau_{k+2}\right)-N\left(\tau_{k+1}\right)}\right\} \\
= & E\left\{(-1)^{N\left(r_{1}\right)+\cdots+N\left(\tau_{k}\right)}\right\} E\left\{(-1)^{N\left(r_{k+2}\right)-N\left(\tau_{k+1}\right)}\right\}
\end{aligned}
$$

since $N\left(\tau_{k+2}\right)-N\left(\tau_{k+1}\right)$ is independent of $N\left(\tau_{1}\right), \cdots, N\left(\tau_{k}\right)$. As in our previous calculation, we get

$$
E\left\{(-1)^{N\left(\tau_{k+2}\right)-N\left(\tau_{k+1}\right)}\right\}=\exp \left[-2 \int_{\tau_{k+1}}^{\tau_{k+2}} a(s) d s\right] .
$$

Thus, we obtain (replacing $\tau_{k+2}$ and $\tau_{k+1}$ by $\tau$ and $\theta$, respectively)

$$
\sigma_{k+2}(t)=\int_{0}^{t} d \tau \int_{0}^{\tau} \exp \left[-2 \int_{\theta}^{\tau} a(s) d s\right] \sigma_{k}(\theta) d \theta
$$


which is just (5), for the case $v(t)=t^{k+2}, k \geqq 0$. Thus, our proof is complete. We note that a similar computation of the moments of $T$, using the Laplace transform (which works only for constant $a$ ) is carried out in [1].

4. Scope of the method. If $L$ is any reasonable linear operator on functions $\phi=\phi(x)$ defined for $x$ in some region $\Omega$ of $E^{n}$, and $v=v(x, t)$ varies continuously with $t$, we may expect that

$$
L E\{v(x, T(t))\}=E\{L v(x, T(t))\} .
$$

Since $E\{\cdot\}$ is in fact given by a Stieltjes integral (see our remark below), this will certainly be true when $L$ is a linear operator taking $D^{\prime}(\Omega)$ (the space of distributions on $\Omega$ ) into itself, continuous in the Schwartz topology. For any such operator, we have at once the following

THEOREM. Let $v(x, t)$ be twice continuously differentiable in $t$ for each $x \in \Omega$ and $t \in(-\tau, \tau)$, and let it satisfy there

$$
\frac{\partial^{2} v}{\partial t^{2}}=L v .
$$

Then $u(x, t)=E\{v(x, T(t))\}$ satisfies

$$
\frac{\partial^{2} u}{\partial t^{2}}+2 a(t) \frac{\partial u}{\partial t}=L u \quad \text { in } \Omega \times(0, \tau)
$$

with

$$
\lim _{t \rightarrow 0+} u(x, t)=v(x, 0)
$$

and

$$
\lim _{t \rightarrow 0+} \frac{\partial u}{\partial t}(x, t)=\frac{\partial v}{\partial t}(x, 0) \quad \text { for } x \in \Omega .
$$

REMARKs. The transformation discussed above can be given a completely nonprobabilistic form. For, we may write

$$
u(x, t)=\int_{-\infty}^{\infty} v(x, s) d_{s} \alpha(s, t)
$$

where $\alpha(s, t)=P\{T(t) \leqq s\}$. (Since $\alpha=0$ for $s \leqq-t$ and $\alpha=1$ for $s \geqq t$ the integral above is really taken between $-t$ and $t$.) But, using the lemma, $\alpha$ is easily seen to be a solution of the simple telegrapher's equation 


$$
\frac{\partial^{2} \alpha}{\partial t^{2}}+2 a(t) \frac{\partial \alpha}{\partial t}=\frac{\partial^{2} \alpha}{\partial s^{2}} \quad \text { for } t>0, \text { all } s
$$

with

$$
\alpha(s, 0)=H_{0}(s)
$$

and

$$
\frac{\partial \alpha}{\partial t}(s, 0)=-\delta_{0}(s)
$$

where $H_{0}(s)$ is the Heaviside unit function with jump at $s=0$, and $\delta_{0}(s)$ is the Dirac distribution centered at $s=0$. The representation of a solution of (7) as an expectation may be of some interest, however, in obtaining a priori estimates; proceeding rather crudely, we observe the following: If $\|\cdot\|$ is any reasonable norm on functions $\phi(x)$ defined on $\Omega$, then

$$
\sup _{0 \leqq t<\tau}\|u\| \leqq \sup _{-\tau \leqq t \leqq \tau}\|v\|,
$$

where $u$ and $v$ are as above. Hopefully, more detailed information may be obtained by exploiting the growth of $a(t)$.

Added in proof. Through conversation with Louis Nirenberg we have been made aware of the following two facts: The transformation above, in its classical form, is an example of a transmutation operator (J. Delsarte, Sur certaines transformations fonctionelles relatives aux équations linéaires aux derivées partielles du second ordre, C. R. Acad. Sci. Paris 206 (1938), 1780-1782). Furthermore, one may deduce that $\alpha(s, t)$ must be a probability distribution from a maximum principle for hyperbolic equations (H. Weinberger, $A$ maximum property of Cauchy's problem, Ann. of Math. (2) 64 (1956), 505-513).

\section{REFERENCES}

1. M. Kac, Some stochastic problems in physics and mathematics, Magnolia Petroleum Company Colloquium Lectures in the Pure and Applied Sciences, No. 2, 1956 (mimeographed lecture notes).

2. S. Goldstein, On diffusion by discontinuous movements and on the telegraph equation, Quart. J. Mech. Appl. Math. 4 (1951), 129-156.

3. E. B. Dynkin, Theory of Markov processes, Prentice-Hall, Englewood Cliffs, N. J., 1961.

The Rockefeller Institute 